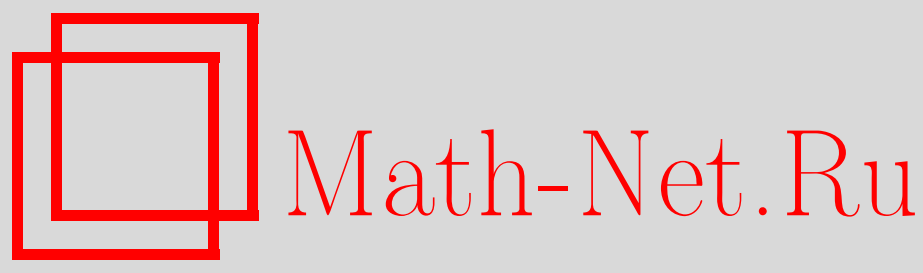

Г. Г. Козлов, Вычисление спектральной зависимости критерия локализации Андерсона в одномерной системе при коррелированном диагональном беспорядке, $Т M \Phi$, 2014, том 179, номер 1, 134-144

DOI: https://doi.org/10.4213/tmf8598

Использование Общероссийского математического портала Math-Net.Ru подразумевает, что вы прочитали и согласны с пользовательским соглашением http://www.mathnet.ru/rus/agreement

Параметры загрузки:

IP : 54.196 .121 .252

26 апреля 2023 г., 12:41:17

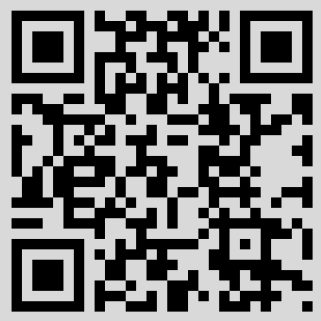




\title{
ВЫЧИСЛЕНИЕ СПЕКТРАЛЬНОЙ ЗАВИСИМОСТИ КРИТЕРИЯ ЛОКАЛИЗАЦИИ АНДЕРСОНА В ОДНОМЕРНОЙ СИСТЕМЕ ПРИ КОРРЕЛИРОВАННОМ ДИАГОНАЛЬНОМ БЕСПОРЯДКЕ
}

\begin{abstract}
Рассмотрена задача о вычислении критерия Андерсона для одномерной разупорядоченной цепочки с коррелированным беспорядком. Для решения указанной задачи развита теория возмущений, в которой малым параметром является обратный радиус корреляции. Показано, что, кроме естественного уменьшения степени локализации в коррелированной системе, имеет место значительное отличие ее спектральной зависимости от спектральной зависимости для некоррелированных цепочек. В расчетах используется метод построения совместной статистики функций Грина, примененный ранее для анализа некоррелированных одномерных систем. Теоретические расчеты иллюстрируются численным экспериментом.
\end{abstract}

Ключевые слова: локализация Андерсона, коррелированный беспорядок, функция Грина.

DOI: $10.4213 / \operatorname{tmf} 8598$

\section{1. ВВЕДЕНИЕ, ПОСТАНОВКА ЗАДАЧИ И ОСНОВНЫЕ РЕЗУЛЬТАТЫ}

Среди математических моделей пространственно-однородных разупорядоченных систем наиболее популярными являются модели, в которых беспорядок представляется некоторым дельта-коррелированным случайным полем. Такие модели являются простейшими, и один из возможных шагов на пути их усложнения состоит в введении корреляций. Вопрос о влиянии корреляций является тем более актуальным, что в реальных системах следует ожидать коррелированного беспорядка. K тому же, как было показано в работах [1]-[3], важнейшее свойство случайных

${ }^{*}$ Научно-исследовательский институт физики им. В. А. Фока, Санкт-Петербургский государственный университет, Санкт-Петербург, Россия.

E-mail: gkozlov@photonics.phys.spbu.ru 
систем - локализация собственных состояний - может существенно зависеть от наличия корреляций.

Для описания локализации в некоррелированных низкоразмерных случайных системах широко используется понятие длины локализации [4]. То же можно сказать и о коррелированных системах [5]-[8]. Несмотря на высокий информативный потенциал понятия длины локализации, применение подобных понятий не всегда позволяет исчерпывающим образом судить о характере (локализованный-делокализованный) собственных состояний случайной системы [9], [10] (см. также работу [5]). По этой причине дополнительное исследование локализации в случайных системах (как коррелированных, так и некоррелированных) с помощью базового критерия Андерсона [4], [11] представляется необходимым.

В настоящей работе мы исследуем локализацию собственных состояний в одномерной коррелированной цепочке, применяя для анализа метод, предложенный Дайсоном [12]. Развитие этого метода дало возможность получить ряд результатов, касающихся как спектральных [13], [14], так и локализационных [15]-[18] свойств одномерных разупорядоченных систем. В указанных работах исследовались некоррелированные случайные цепочки, известным исключением можно считать работу [16], где была проанализирована случайная цепочка со сложной структурной единицей, эта цепочка может (правда, довольно искусственно) рассматриваться как коррелированная.

В настоящей работе для простейшей диагонально разупорядоченной коррелированной одномерной цепочки выполнен расчет спектральной зависимости степени локализации в смысле критерия Андерсона и продемонстрировано существенное отличие указанной зависимости от случая некоррелированной цепочки. Для расчета используется предложенный в работах [15]-[17] метод, основанный на построении совместной статистики опережающей и запаздывающей функций Грина. В приводимом ниже расчете параметром малости является обратный радиус корреляции, и полученные формулы работают при значительной (по отношению к невозмущенному гамильтониану) величине разупорядочения, когда обычная квантово-механическая теория возмущений неприменима. Поэтому полученные результаты еще раз демонстрируют эффективность и универсальность предложенного в работах [15]-[17] метода совместной статистики функций Грина, который позволяет проанализировать целый ряд задач, некоторые из них (например, задача Ллойда [17]) являются квантовомеханически непертурбативными.

Перейдем к постановке задачи. Матрица гамильтониана разупорядоченной цепочки длины $N$, которую мы предполагаем исследовать, имеет в узельном представлении стандартный вид [4] (диагональный беспорядок)

$$
H_{n, n^{\prime}}=\delta_{n, n^{\prime}} \varepsilon_{n}+\delta_{n, n^{\prime}+1}+\delta_{n, n^{\prime}-1}, \quad n, n^{\prime}=1, \ldots, N
$$

однако, в отличие от традиционной постановки задачи, случайные узельные энергии $\varepsilon_{n}$ образуют теперь коррелированную последовательность со следующим простейшим механизмом корреляции, сходным с описанным, например, в работе [5]. Сопоставим каждому узлу $n$ нашей цепочки некоторую вспомогательную случайную величину $\xi_{n}$, причем величины $\xi_{n}, n=1, \ldots, N$, будем считать взаимно независимыми и имеющими заданную плотность распределения $P(x)$. С помощью этих 
величин построим узельные энергии $\varepsilon_{n}$ следующим образом:

$$
\varepsilon_{n}=\left(1-e^{-\alpha}\right) \sum_{m \leqslant n} e^{\alpha(m-n)} \xi_{m}, \quad \alpha>0 .
$$

При таком построении величины $\varepsilon_{n}, n=1, \ldots, N$, будут коррелированы, причем корреляционная функция $\left\langle\varepsilon_{n} \varepsilon_{n^{\prime}}\right\rangle$ имеет экспоненциальный вид:

$$
\left\langle\varepsilon_{n} \varepsilon_{n^{\prime}}\right\rangle=\left\langle\xi^{2}\right\rangle\left(\frac{1-e^{-\alpha}}{1+e^{-\alpha}}\right) e^{-\alpha\left|n-n^{\prime}\right|}=\left\langle\xi^{2}\right\rangle\left(\frac{1-\beta}{1+\beta}\right) \beta^{\left|n-n^{\prime}\right|}, \quad \beta \equiv e^{-\alpha}, \quad \beta \in[0,1],
$$

с характерным радиусом корреляции $R=1 / \alpha$. Отметим, что последовательность $\varepsilon_{n}, n=1, \ldots, N$, причинно коррелирована, т. е. случайная величина $\varepsilon_{n}$ зависит только от тех $\xi_{m}$, у которых $m \leqslant n$. Для дальнейших рассуждений существенным является следующее проверяемое прямым расчетом соотношение:

$$
\varepsilon_{n+1}=\beta \varepsilon_{n}+(1-\beta) \xi_{n+1} .
$$

Ниже мы будем считать плотность распределения вспомогательных независимых величин $\xi_{n}$ гауссовой,

$$
P(x)=\frac{1}{\Delta} \frac{1}{\sqrt{\pi}} e^{-(x / \Delta)^{2}} .
$$

Нетрудно убедиться в том, что в этом случае плотность распределения $\mathcal{P}(z)$ узельных энергий $\varepsilon_{n}$ также будет гауссовой,

$$
\mathcal{P}(z)=\frac{1}{\sqrt{\pi}} \frac{1}{\bar{\Delta}} e^{-(z / \bar{\Delta})^{2}}, \quad \text { где } \quad \bar{\Delta} \equiv \Delta \sqrt{\frac{1-\beta}{1+\beta}} .
$$

Для такой цепочки с коррелированным диагональным беспорядком рассмотрим стандартные [4], [15]-[17] задачи вычисления критерия Андерсона $D$ - остаточной плотности возбуждения на краевом узле цепочки (о связи параметра $D$ с областью локализации см. работу [18]) - и нахождения спектральной зависимости степени локализации $W(U)$ [15]. Указанные величины выражаются через собственные числа $E_{\lambda}$ и краевые компоненты собственных векторов $\Psi^{\lambda}$ матрицы (1) следующим образом:

$$
W(U) d U=\left\langle\sum_{E_{\lambda} \in[U, U+d U]}\left|\Psi_{N}^{\lambda}\right|^{4}\right\rangle, \quad D=\int W(U) d U .
$$

В соответствии с методикой, предложенной в работах [15]-[17], для решения поставленной задачи необходимо знать совместную статистику двух краевых функций Грина $(\mathrm{К} Г) g_{N}\left(\Omega_{1}\right)$ и $g_{N}\left(\Omega_{2}\right)$ при разных (комплексных) энергиях $\Omega_{1}=U_{1}+i V_{1}$ и $\Omega_{2}=U_{2}-i V_{2}$, причем $V_{1,2}>0$. Правила вычисления критерия Андерсона $D$ и функции $W(U)$ с помощью этой статистики описаны в работах [15]-[17]. Ниже получены соответствующие формулы, учитывающие коррелированный характер узельных энергий. Сразу отметим, что, в отличие от задач, проанализированных в работах [15]-[17], в рассматриваемом случае коррелированного беспорядка мы можем получить замкнутое уравнение только для совместной статистики двух краевых функций Грина $g_{N}\left(\Omega_{1,2}\right)$ и энергии краевого узла $\varepsilon_{N}$. Указанная статистика 
представляет собой функцию $\rho\left(z_{1}, z_{2}, \varepsilon\right)$ двух комплексных и одного вещественного аргументов и определяется так, что величина $\rho\left(x_{1}+i y_{1}, x_{2}+i y_{2}, \varepsilon\right) d x_{1} d y_{1} d x_{2} d y_{2} d \varepsilon$ равна вероятности совместного наступления событий

$$
\begin{array}{cc}
\operatorname{Re} g_{N}\left(\Omega_{1}\right) \in\left[x_{1}, x_{1}+d x_{1}\right], & \operatorname{Im} g_{N}\left(\Omega_{1}\right) \in\left[y_{1}, y_{1}+d y_{1}\right], \\
\operatorname{Re} g_{N}\left(\Omega_{2}\right) \in\left[x_{2}, x_{2}+d x_{2}\right], & \operatorname{Im} g_{N}\left(\Omega_{2}\right) \in\left[y_{2}, y_{2}+d y_{2}\right], \\
\varepsilon_{N} \in[\varepsilon, \varepsilon+d \varepsilon] . &
\end{array}
$$

Наша первая задача - вывод замкнутого уравнения для функции $\rho\left(z_{1}, z_{2}, \varepsilon\right)$. Для этого мы, как и в работах [15]-[17], используем известное (см., например, книгу [4]) соотношение, позволяющее выразить КФГ $g_{N+1}(\Omega)$ цепочки с одним добавленным узлом через КФГ $g_{N}(\Omega)$ исходной цепочки:

$$
g_{N+1}(\Omega)=\frac{1}{\Omega-\varepsilon_{N+1}-g_{N}(\Omega)} .
$$

Для компактификации расчетов нам будет удобно использовать следующее определение дельта-функции, аргументом которой является аналитическая функция $F(z)=u(x, y)+i v(x, y)$ комплексной переменной $z=x+i y$ :

$$
\delta[F(z)] \equiv \delta[u(x, y)] \delta[v(x, y)] .
$$

Такая дельта-функция всегда вещественна и может быть преобразована следующим образом. Пусть $z_{0}=x_{0}+i y_{0}$ есть корень функции $F(z)$. Тогда прямой расчет с использованием соотношений Коши-Римана для функции $F(z)$ показывает, что

$$
\delta[F(z)]=\frac{\delta\left[z-z_{0}\right]}{\left|F^{\prime}\left(z_{0}\right)\right|^{2}}, \quad F\left(z_{0}\right)=0,
$$

причем стоящая в числителе дельта-функция комплексной функции $z-z_{0}$ понимается в смысле (7).

Также для сокращения записи мы обозначим символом $\int_{R} d z$ следующие интегралы от функций (необязательно аналитических) $f(z)=\tilde{u}(x, y)+i \tilde{v}(x, y)$ комплексной переменной $z=x+i y$ :

$$
\int_{R} f(z) d z \equiv \iint_{-\infty}^{+\infty}[\tilde{u}(x, y)+i \tilde{v}(x, y)] d x d y .
$$

Вернемся к выводу уравнения для совместной статистики $\rho\left(z_{1}, z_{2}, \varepsilon\right)$ и добавим к нашей цепочке из $N$ узлов еще один узел: $N \rightarrow N+1$. Если обозначить через $\tilde{\rho}\left(z_{1}, z_{2}, \varepsilon\right)$ функцию, аналогичную $\rho$, только описывающую совместную статистику КФГ цепочки с добавленным узлом, то с учетом введенных определений и соотношений (2) и (6) для нее можно написать следующее выражение:

$$
\begin{aligned}
& \tilde{\rho}\left(z_{1}, z_{2}, \varepsilon\right)= \\
& =\left\langle\delta\left(z_{1}-\frac{1}{\Omega_{1}-\varepsilon_{N+1}-g_{N}\left(\Omega_{1}\right)}\right) \delta\left(z_{2}-\frac{1}{\Omega_{2}-\varepsilon_{N+1}-g_{N}\left(\Omega_{2}\right)}\right) \delta\left(\varepsilon-\varepsilon_{N+1}\right)\right\rangle= \\
& =\left\langle\delta\left(z_{1}-\frac{1}{\Omega_{1}-\varepsilon-g_{N}\left(\Omega_{1}\right)}\right) \delta\left(z_{2}-\frac{1}{\Omega_{2}-\varepsilon-g_{N}\left(\Omega_{2}\right)}\right) \delta\left(\varepsilon-\beta \varepsilon_{N}-(1-\beta) \xi_{N+1}\right)\right\rangle=
\end{aligned}
$$




$$
\begin{aligned}
= & \int_{R} d z_{1}^{\prime} d z_{2}^{\prime} \int P(x) \rho\left(z_{1}^{\prime} z_{2}^{\prime}, \varepsilon^{\prime}\right) \times \\
& \times \delta\left(z_{1}-\frac{1}{\Omega_{1}-\varepsilon-z_{1}^{\prime}}\right) \delta\left(z_{2}-\frac{1}{\Omega_{2}-\varepsilon-z_{2}^{\prime}}\right) \delta\left(\varepsilon-\beta \varepsilon^{\prime}-(1-\beta) x\right) d \varepsilon^{\prime} d x .
\end{aligned}
$$

Как обычно, при $N \rightarrow \infty$ (термодинамический предел) должно выполняться равенство $\rho=\tilde{\rho}$ (условие стационарности статистики). Используя формулу (8), проводем интегрирования с дельта-функциями и получим для стационарной совместной функции распределения $\rho\left(z_{1}, z_{2}, \varepsilon\right)$ следующее замкнутое уравнение:

$$
\beta\left|z_{1}\right|^{4}\left|z_{2}\right|^{4} \rho\left(z_{1}, z_{2}, \varepsilon\right)=\int P(x) \rho\left(\Omega_{1}-\varepsilon-\frac{1}{z_{1}}, \Omega_{2}-\varepsilon-\frac{1}{z_{2}}, \frac{\varepsilon-(1-\beta) x}{\beta}\right) d x .
$$

Для вычисления остаточной плотности $D$ и функции $W(U)$ требуются функции Грина с предельно малыми и имеющими разные знаки мнимыми частями энергетических аргументов. В полной аналогии с тем, как это было сделано в работах [15]-[17], данное обстоятельство можно использовать для сведения задачи к анализу уравнения для совместной статистики вещественных КФГ и энергии крайнего узла, которое имеет вид

$$
\beta x_{1}^{2} x_{2}^{2} \sigma\left(x_{1}, x_{2}, \varepsilon\right)=\int P(x) \sigma\left(U_{1}-\varepsilon-\frac{1}{x_{1}}, U_{2}-\varepsilon-\frac{1}{x_{2}}, \frac{\varepsilon-(1-\beta) x}{\beta}\right) d x .
$$

Дальнейшие вычисления также производятся путем прямого обобщения результатов и формул работ [15]-[17], в которых показано, что для вычисления функции $W(U)$ необходима только сингулярная по разности энергетических аргументов $U_{2}-U_{1} \equiv \omega$ часть функции $\sigma\left(x_{1}, x_{2}, \varepsilon\right)$, причем эта сингулярность имеет вид полюса. Таким образом, при малых $\omega$ функция $\sigma\left(x_{1}, x_{2}, \varepsilon\right) \approx \operatorname{sing} \sigma\left(x_{1}, x_{2}, \varepsilon\right)$, где $\operatorname{sing} \sigma\left(x_{1}, x_{2}, \varepsilon\right)$ может быть записана в виде

$$
\operatorname{sing} \sigma\left(x_{1}, x_{2}, \varepsilon\right)=\frac{1}{\omega} \mathcal{F}_{U}\left(x_{1}, x_{2}, \varepsilon\right)
$$

Здесь мы сделали переобозначение $U_{1} \rightarrow U$. Если известна функция $\mathcal{F}_{U}\left(x_{1}, x_{2}, \varepsilon\right)$, то функция $W(U)$ и критерий Андерсона $D$ могут быть рассчитаны по формулам

$$
W(U)=\lim _{a \rightarrow \infty} a^{2} \int \mathcal{F}_{U}(x, a, \varepsilon) d x d \varepsilon, \quad D=\int W(U) d U .
$$

Вывод формул (9)-(11) полностью аналогичен выводу соответствующих формул из работ [15]-[17].

Таким образом, задача сводится к нахождению сингулярной части $\mathcal{F}\left(x_{1}, x_{2}, \varepsilon\right) / \omega$ решения уравнения (9). В следующем разделе для решения этой задачи будет развита теория возмущений, в которой параметром малости является обратный радиус корреляции $1 / R=-\ln \beta$, и получены следующие выражения для спектральной зависимости степени локализации $W(U)$ и критерия Андерсона $D$ :

$$
\begin{gathered}
W(U)=\frac{\bar{\Delta}^{2}}{4 \pi R} \operatorname{Re} \int \frac{\mathcal{P}(\varepsilon)}{\sqrt{4-(U-\varepsilon)^{2}}} d \varepsilon+O\left(\frac{1}{R^{2}}\right), \\
D=\int W(U) d U=\frac{\bar{\Delta}^{2}}{4 R}+O\left(\frac{1}{R^{2}}\right) .
\end{gathered}
$$


Эти выражения применимы для коррелированной диагонально разупорядоченной цепочки при больших радиусах корреляции $R \gg 1$. Обратим внимание на то, что эти формулы могут работать и тогда, когда $\bar{\Delta}>1$, т. е. при значительных величинах беспорядка.

\section{2. ПРИБЛИЖЕНИЕ БОЛЬШИХ РАДИУСОВ КОРРЕЛЯЦИИ}

Пусть радиус корреляции $R \gg 1$ и, следовательно, $\beta \approx 1$. Чтобы найти приближенное решение уравнения (9), построим теорию возмущений по малому параметру $y \equiv 1-\beta>0(y \approx 1 / R)$. Будем считать, что плотность распределения энергий узлов имеет вид (4), причем $\bar{\Delta}$ фиксировано и необязательно мало. Это означает, что ширина $\Delta$ распределения (3) вспомогательных величин $\xi_{n}$ будет увеличиваться при $\beta \rightarrow 1$. Таким образом, плотность распределения $P(x)$ вспомогательных величин $\xi_{n}$ зависит от $\beta$ и имеет вид (3), где

$$
\Delta=\bar{\Delta} \sqrt{\frac{1+\beta}{1-\beta}}
$$

Для дальнейших рассуждений удобно перейти в уравнении (9) к новой функции $\mathcal{R}\left(x_{1}, x_{2}, \varepsilon\right)$ по формуле

$$
\mathcal{R}\left(U_{1}-\varepsilon-x_{1}, U_{2}-\varepsilon-x_{2}, \beta \varepsilon\right) \equiv \sigma\left(x_{1}, x_{2}, \varepsilon\right) .
$$

Тогда из $(9)$ для функции $\mathcal{R}\left(x_{1}, x_{2}, \varepsilon\right)$ получается следующее уравнение:

$$
\beta \frac{\mathcal{R}\left(U_{1}-\varepsilon-1 / x_{1}, U_{2}-\varepsilon-1 / x_{2}, \beta \varepsilon\right)}{x_{1}^{2} x_{2}^{2}}=\int P(x) \mathcal{R}\left(x_{1}, x_{2}, \varepsilon-y x\right) d x .
$$

Рассмотрим интеграл в правой части этого уравнения. Функция $P(x)$ существенно отлична от нуля при $x \in[-\Delta, \Delta]$, а размер соответствующей области, в которой меняется фигурирующая в (15) величина $y x$, оценивается как

$$
\Delta y=\bar{\Delta} \sqrt{1-\beta^{2}} \approx \bar{\Delta} \sqrt{2 y} \rightarrow 0 \quad \text { при } \quad \beta \rightarrow 1 .
$$

Поэтому при малом $y$ функцию $\mathcal{R}\left(x_{1}, x_{2}, \varepsilon-y x\right)$ в правой части (15) можно разложить в степенной ряд до второго члена (первый член отсутствует, поскольку первый момент функции $P(x)$ равен нулю):

$$
\beta \frac{\mathcal{R}\left(U_{1}-\varepsilon-1 / x_{1}, U_{2}-\varepsilon-1 / x_{2}, \beta \varepsilon\right)}{x_{1}^{2} x_{2}^{2}}=\mathcal{R}\left(x_{1}, x_{2}, \varepsilon\right)+\frac{\partial^{2} \mathcal{R}\left(x_{1}, x_{2}, \varepsilon\right)}{\partial \varepsilon^{2}} \frac{y^{2} \Delta^{2}}{4} .
$$

Здесь мы воспользовались тем, что для функции $(3) \int x^{2} P(x) d x=\Delta^{2} / 2$. Подставляя $\Delta$ из (13) и учитывая, что $\beta \approx 1$, получаем

$$
\beta \frac{\mathcal{R}\left(U_{1}-\varepsilon-1 / x_{1}, U_{2}-\varepsilon-1 / x_{2}, \beta \varepsilon\right)}{x_{1}^{2} x_{2}^{2}}=\mathcal{R}\left(x_{1}, x_{2}, \varepsilon\right)+\frac{\partial^{2} \mathcal{R}\left(x_{1}, x_{2}, \varepsilon\right)}{\partial \varepsilon^{2}} \frac{y \bar{\Delta}^{2}}{2} .
$$

Теперь примем во внимание, что функция $\mathcal{R}$ непосредственно зависит от $y$. В линейном приближении эта зависимость имеет вид

$$
\mathcal{R}\left(x_{1}, x_{2}, \varepsilon\right)=\mathcal{R}_{0}\left(x_{1}, x_{2}, \varepsilon\right)+y \mathcal{R}_{1}\left(x_{1}, x_{2}, \varepsilon\right)+\cdots .
$$


Подставим это выражение в (16) и приравняем коэффициенты при равных степенях $y$. Приравнивание коэффициентов при $y^{0}$ дает

$$
\frac{\mathcal{R}_{0}\left(U_{1}-\varepsilon-1 / x_{1}, U_{2}-\varepsilon-1 / x_{2}, \varepsilon\right)}{x_{1}^{2} x_{2}^{2}}=\mathcal{R}_{0}\left(x_{1}, x_{2}, \varepsilon\right) .
$$

Учитывая это уравнение и приравнивая коэффициенты при $y$, получаем

$$
\left[\mathcal{H}_{U_{1}-\varepsilon}\left(x_{1}\right) \mathcal{H}_{U_{2}-\varepsilon}\left(x_{2}\right)-1\right] \mathcal{R}_{1}\left(x_{1}, x_{2}, \varepsilon\right)=\mathcal{A},
$$

где

$\mathcal{A} \equiv \frac{\bar{\Delta}^{2}}{2} \frac{\partial^{2} \mathcal{R}_{0}\left(x_{1}, x_{2}, \varepsilon\right)}{\partial \varepsilon^{2}}+\left.\frac{\varepsilon}{x_{1}^{2} x_{2}^{2}} \frac{\partial \mathcal{R}_{0}\left(U_{1}-\varepsilon-1 / x_{1}, U_{2}-\varepsilon-1 / x_{2}, \theta\right)}{\partial \theta}\right|_{\theta=\varepsilon}+\mathcal{R}_{0}\left(x_{1}, x_{2}, \varepsilon\right)$.

В уравнении (18) мы обозначили через $\mathcal{A}$ правую часть уравнения для $\mathcal{R}_{1}$ и ввели функциональный оператор $\mathcal{H}_{U}(x)$ [15], [19], действующий на произвольную функцию $f(x)$ по следующему правилу: $\mathcal{H}_{U}(x) f(x) \equiv f(U-1 / x) / x^{2}$. Известно [15], [19], что собственная функция этого оператора, соответствующая единичному собственному числу, есть лоренциан $\mathcal{L}_{U}(x)$, явное выражение для которого приведено ниже (см. формулы (24)). Имея это в виду, нетрудно убедиться в том, что решение $\mathcal{R}_{0}$ уравнения (17) имеет следующий вид:

$$
\mathcal{R}_{0}\left(x_{1}, x_{2}, \varepsilon\right)=\mathcal{L}_{U_{1}-\varepsilon}\left(x_{1}\right) \mathcal{L}_{U_{2}-\varepsilon}\left(x_{2}\right) \mathcal{P}(\varepsilon)
$$

где $\mathcal{P}(\varepsilon)$ есть плотность распределения узельных энергий в форме (4).

Чтобы решить функциональное уравнение (18) для первой неисчезающей поправки $\mathcal{R}_{1}$, разложим правую и левую части этого уравнения по собственным функциям $\sigma_{U_{1}-\varepsilon}^{n}\left(x_{1}\right) \sigma_{U_{2}-\varepsilon}^{m}\left(x_{2}\right)$ оператора $\mathcal{H}_{U_{1}-\varepsilon}\left(x_{1}\right) \mathcal{H}_{U_{2}-\varepsilon}\left(x_{2}\right)$, как это сделано в работах [15]-[17], и приравняем соответствующие коэффициенты. Разложение для $\mathcal{R}_{1}$ имеет вид

$$
\mathcal{R}_{1}\left(x_{1}, x_{2}, \varepsilon\right)=\sum_{m, n} C_{n m} \sigma_{U_{1}-\varepsilon}^{n}\left(x_{1}\right) \sigma_{U_{2}-\varepsilon}^{m}\left(x_{2}\right)
$$

коэффициенты $C_{n m}$ следует определить, разложив по функциям $\sigma_{U_{1}-\varepsilon}^{n}\left(x_{1}\right) \sigma_{U_{2}-\varepsilon}^{m}\left(x_{2}\right)$ известную правую часть уравнения (18) и приравняв соответствующие коэффициенты. Чтобы это сделать, напомним полученные в статье [19] явные выражения для собственных функций $\sigma_{U}^{n}(x)$ и собственных значений $\lambda_{n}$ оператора $\mathcal{H}_{U}(x)$ при $|U|<2$ :

$$
\begin{gathered}
\sigma_{U}^{n}(x)=\mathcal{L}_{U}(x)\left(\frac{R_{U}^{*}-x}{R_{U}-x}\right)^{n} \equiv \mathcal{L}_{U}(x) G_{U}^{n}(x), \\
\lambda_{n}=\left(\frac{U+i \sqrt{4-U^{2}}}{U-i \sqrt{4-U^{2}}}\right)^{n}, \quad\left|\lambda_{n}\right|=1,
\end{gathered}
$$

где

$$
\begin{gathered}
G_{U}(x) \equiv \frac{R_{U}^{*}-x}{R_{U}-x}, \quad R_{U} \equiv \frac{U+i \sqrt{4-U^{2}}}{2}, \\
\mathcal{L}_{U}(x) \equiv \frac{1}{2 \pi i}\left(\frac{1}{x-R_{U}}-\frac{1}{x-R_{U}^{*}}\right)
\end{gathered}
$$


Напомним также правило проецирования на систему функций (22), которое заключается в том, что произвольная функция $f(x)$ может быть представлена в виде ряда:

$$
f(x)=\sum_{n=-\infty}^{+\infty} A_{n} \sigma_{U}^{n}(x), \quad \text { где } \quad A_{n}=\int \frac{f(x)}{G_{U}^{n}(x)} d x .
$$

Из уравнения (18) следует, что $\int \mathcal{A} d x_{1} d x_{2}=0$. Это приводит к тому, что функция $\mathcal{P}(\varepsilon)(4)$ должна удовлетворять уравнению

$$
\frac{\bar{\Delta}^{2}}{2} \frac{d^{2} \mathcal{P}}{d \varepsilon^{2}}+\varepsilon \frac{d \mathcal{P}}{d \varepsilon}+\mathcal{P}(\varepsilon)=0
$$

которое получается из правой части уравнения (18), если в нее подставить $\mathcal{R}_{0}$ в форме $(20)$ и проинтегрировать по $x_{1}$ и $x_{2}$. Прямая проверка показывает, что уравнение (25) действительно выполняется.

Имея все это в виду, получаем для коэффициентов $C_{n m}$ (кроме $C_{00}$, который определяется нормировкой и не является сингулярным) в формуле (21) следующие выражения:

$$
C_{n m}=\frac{A_{n m}}{\lambda_{n}\left(U_{1}-\varepsilon\right) \lambda_{m}\left(U_{2}-\varepsilon\right)-1}, \quad A_{n m}=\int \frac{\mathcal{A}\left(x_{1}, x_{2}\right)}{G_{U_{1}-\varepsilon}^{n}\left(x_{1}\right) G_{U_{2}-\varepsilon}^{m}\left(x_{2}\right)} d x_{1} d x_{2} .
$$

Вспомним теперь, что нам необходима лишь сингулярная по $\omega=U_{2}-U_{1}$ часть оператора $\mathcal{R}_{1}$, которой соответствуют члены разложения с $n=-m[15]$ : именно у этих членов в коэффициентах (26) знаменатель обращается в нуль при $U_{1}=U_{2}$. Для малых $\omega$ справедливо разложение

$$
1-\lambda_{n}\left(U_{1}-\varepsilon\right) \lambda_{-n}\left(U_{1}+\omega-\varepsilon\right)=-\frac{2 i n \omega}{\sqrt{4-\left(U_{1}-\varepsilon\right)^{2}}}+O\left(\omega^{2}\right) .
$$

В интересующую нас сингулярную часть дает вклад только первое слагаемое в выражении (19) для $\mathcal{A}$, и мы приведем расчет только для этого слагаемого ${ }^{1)}$. Введем обозначение $a_{n} \equiv A_{n,-n}$ для соответствующих коэффициентов разложения. На этом этапе расчета уже можно приравнять энергетические аргументы, $U_{1}=U_{2} \equiv U$, поскольку сингулярность определяется только знаменателем коэффициентов (26) с $n=-m$. Имеем

$$
a_{n}=\left.\frac{\bar{\Delta}^{2}}{2} \frac{d^{2}}{d \theta^{2}}\left(\int \frac{\mathcal{R}_{0}\left(x_{1}, x_{2}, \theta\right)}{G_{U-\varepsilon}^{n}\left(x_{1}\right) G_{U-\varepsilon}^{-n}\left(x_{2}\right)} d x_{1} d x_{2}\right)\right|_{\theta=\varepsilon} .
$$

Как и в работах [15]-[17], определим величины $J_{n}(\theta)$ следующим образом:

$$
J_{n}(\theta) \equiv \int \frac{\mathcal{L}_{U-\theta}(x)}{G_{U-\varepsilon}^{n}(x)} d x, \quad J_{-n}(\theta)=J_{n}^{*}(\theta), \quad J_{n}(\varepsilon)=0 \quad \text { при } \quad n \neq 0 .
$$

Используя выражения (23) и интегрируя с помощью теоремы о вычетах, можно показать, что

$$
J_{n}(\theta)=\left(\frac{R_{U-\varepsilon}-R_{U-\theta}}{R_{U-\varepsilon}^{*}-R_{U-\theta}}\right)^{n}, \quad n>0 .
$$

1) Проведя расчеты по приведенной ниже схеме, можно убедиться в том, что остальные слагаемые в выражении (19) не дают вклада. 
Тогда

$$
a_{n}=\left.\frac{\bar{\Delta}^{2}}{2} \frac{d^{2}\left(\left|J_{n}(\theta)\right|^{2} \mathcal{P}(\theta)\right)}{d \theta^{2}}\right|_{\theta=\varepsilon},
$$

и для сингулярной части функции $\sigma\left(x_{1}, x_{2}, \varepsilon\right)$ (обозначим ее символом "sing") получаем выражение вида $(10)^{2)}$

$$
\operatorname{sing} \sigma\left(x_{1}, x_{2}, \varepsilon\right)=\frac{1}{\omega} \mathcal{F}_{U}\left(x_{1}, x_{2}, \varepsilon\right),
$$

где

$$
\mathcal{F}_{U}\left(x_{1}, x_{2}, \varepsilon\right)=-\frac{i y}{2} \sqrt{4-(U-\varepsilon)^{2}} \sum_{n \neq 0} \frac{a_{n}}{n} \sigma_{U-\varepsilon}^{n}\left(U-\varepsilon-x_{1}\right) \sigma_{U-\varepsilon}^{-n}\left(U-\varepsilon-x_{2}\right)
$$

и величины $a_{n}$ определяются формулой (28). Отличием $\beta$ от единицы в функциях $\sigma$ и в коэффициентах $A_{n,-n}=a_{n}$ можно пренебречь (они зависят от $\varepsilon$, следовательно, надо взять их значения в точке $\beta \varepsilon$ при переходе от $\mathcal{R}\left(x_{1}, x_{2}, \varepsilon\right)$ к $\sigma\left(x_{1}, x_{2}, \varepsilon\right)$ по формуле (14)). Прямой расчет показывает, что ненулевыми будут только члены суммы (30)) с $n= \pm 1$. Используя соотношения (28) и (29), получаем для $a_{1}=a_{-1}$ следующее выражение:

$$
a_{1}=\mathcal{P}(\varepsilon) \frac{\bar{\Delta}^{2}}{\left[4-(U-\varepsilon)^{2}\right]^{2}} .
$$

Используя доказанные в статье [15] свойства функций (22)

$$
\int \sigma_{U}^{n}(x) x d x=\frac{i}{2} \frac{n}{|n|} \sqrt{4-U^{2}}, \quad \lim _{a \rightarrow \infty} \sigma_{U}^{-n}(a) a^{2}=\frac{\sqrt{4-U^{2}}}{2 \pi}
$$

и вспоминая, что $y=1 / R$ (где $R$ - радиус корреляции, который мы предполагаем большим), с помощью формул (11) получаем для $W(U)$ и остаточной плотности $D$ выражения (12).

Численная проверка формул (12) с помощью соотношений (5) несколько затрудняется тем, что при больших радиусах корреляции $R$ размеры случайных матриц (1) следует брать примерно в $R$ раз бо́льшими, чем при проверке аналогичных формул из работ [15], [17]. Результаты численной проверки приведены на рис. 1. Рис. 1а получен при следующих значениях параметров: $\bar{\Delta}=0.5$ и $R=5$. Вследствие небольшого значения радиуса корреляции рис. 1а демонстрирует лишь качественное соответствие между теорией и численным экспериментом. Из рис. 1б, который получен при $\bar{\Delta}=5$ и $R=50$, видно, что увеличение радиуса корреляции приводит к улучшению согласия теоретической и численной зависимостей, так как формулы (12) получены в приближении больших значений радиуса корреляции.

\section{3. ЗАКЛЮЧЕНИЕ}

В настоящей работе продемонстрировано, что предложенный в работах [15]-[17] метод анализа локализации в одномерных системах, использующий построение совместной статистики опережающей и запаздывающей функций Грина, может быть обобщен на случай коррелированных низкоразмерных разупорядоченных систем.

\footnotetext{
2)Эта часть связана с сингулярной частью функции $\mathcal{R}$ заменой переменных (14).
} 


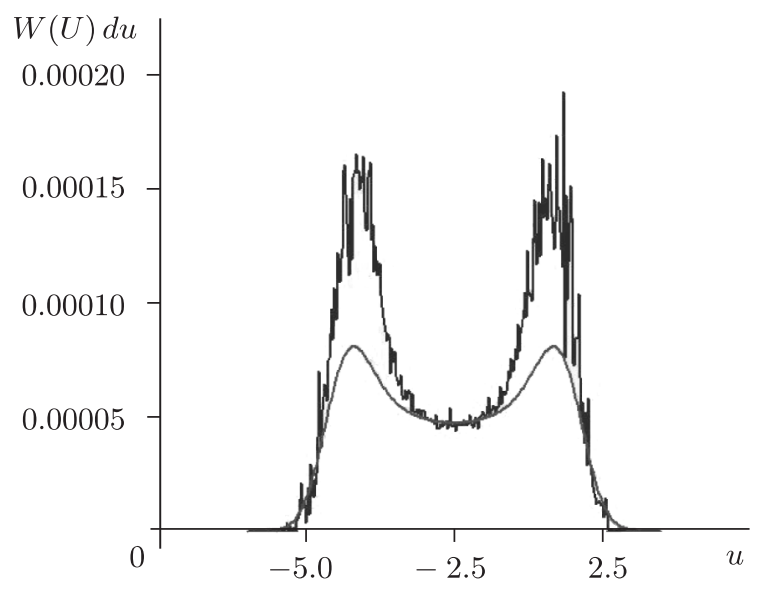

a

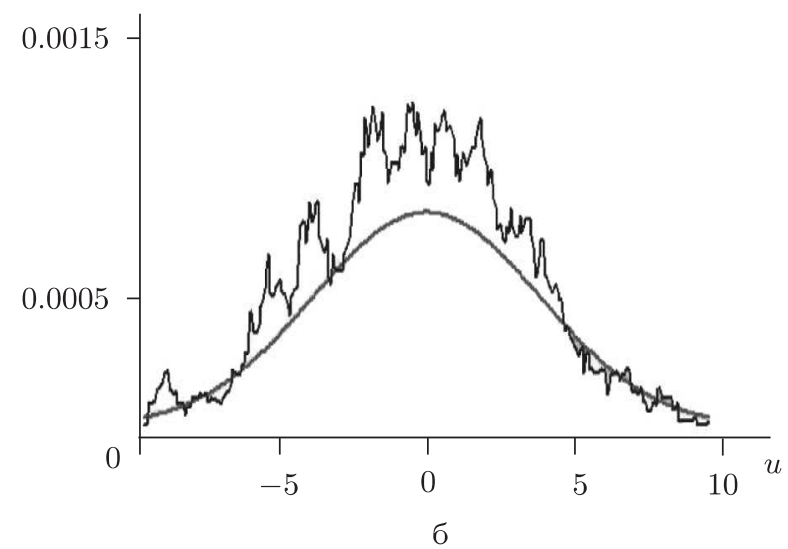

Рис. 1. Спектральные зависимости степени локализации в одномерных коррелированных цепочках при различных значениях степени разупорядочения и радиусов корреляции: при $\bar{\Delta}=0.5$ и $R=5$ для 3000 реализаций (а) и при $\bar{\Delta}=5$ и $R=50$ для 200 реализаций (б). Размер случайной матрицы во всех случаях выбирался равным 2000. Зашумленные графики соответствуют компьютерному эксперименту, гладкие получены с помощью формулы (12) без какой-либо подгонки. При увеличении радиуса корреляции согласие теории и эксперимента улучшается, несмотря на рост степени разупорядочения.

В рамках этого метода оказалось возможным построить простую теорию возмущений для статистики функций Грина, в которой параметром малости является обратный радиус корреляции $1 / R$, и показать, что остаточная плотность $D$ в краевом узле (критерий Андерсона) уменьшается в $R$ раз по сравнению со своим значением для некоррелированной цепочки [15]. Этот результат представляется естественным: исходно созданное возбуждение на краевом узле заведомо рассосредотачивается в области, размер которой имеет порядок радиуса корреляции. 
Более неожиданным представляется второй результат работы, заключающийся в том, что спектральная зависимость $W(U)$ степени локализации, заданная в (12), существенно отличается от спектральной зависимости для некоррелированных цепочек [15] и представляет собой сглаженную плотность состояний упорядоченной цепочки.

Благодарности. Работа выполнена при финансовой поддержке Министерства образования и науки РФ (контракт № 11.G34.31.0067 с Санкт-Петербургским государственным университетом и ведущим ученым А. В. Кавокиным).

\section{Список литературы}

[1] V.A. Malyshev, A. Rodriguez, F. Dominguez-Adame, Phys. Rev. B, 60:20 (1999), 14140-14146.

[2] F. A. B. F. de Moura, M. L. Lyra, Phys. Rev. Lett., 81:17 (1998), 3735-3738.

[3] D. H. Dunlap, H-L. Wu, P. W. Phillips, Phys. Rev. Lett., 65:1 (1990), 88-91.

[4] И. М. Лифшиц, С.А. Гредескул, Л. А. Пастур, Введение в теорию неупорядоченных систем, Наука, М., 1982.

[5] F. M. Izrailev, A. A. Krokhin, N. M. Makarov, Phys. Rep., 512:3 (2012), 125-254, arXiv: 1110.1762 .

[6] M. Titov, H. Schomerus, Phys. Rev. Lett., 95:12 (2005), 126602, 4 pp.

[7] L. I. Deych, M. V. Erementchouk, A. A. Lisyansky, Physica B, 338:1-4 (2003), 79-81.

[8] A. Croy, P. Cain, M. Schreiber, Europ. Phys. J. B, 82:2 (2011), 107-112.

[9] G. Theodorou, M. H. Cohen, Phys. Rev. B, 13:10 (1976), 4597-4601.

[10] G. G. Kozlov, The watching operators method in the theory of Frenkel exciton. Novel criterion of localization and its exact calculation for the non diagonal disordered $1 D$ chain's zero-state, arXiv: cond-mat/9909335.

[11] P. W. Anderson, Phys. Rev., 109:5 (1958), 1492-1505.

[12] F. J. Dyson, Phys. Rev., 92:6 (1953), 1331-1338.

[13] H. Schmidt, Phys. Rev., 105:2 (1957), 425-441.

[14] Г. Г. Козлов, ТМФ, 140:2 (2004), 337-352.

[15] Г. Г. Козлов, ТМФ, 162:2 (2010), 285-303.

[16] G. G. Kozlov, Appl. Math., 2:8 (2011), 965-974.

[17] Г. Г. Козлов, ТМФ, 171:1 (2012), 124-134.

[18] G. G. Kozlov, Spectral dependence of degree of localization of eigenfunctions of the $1 D$ Schrodinger equation with a peacewise-constant random potential, arXiv: 1105.2678.

[19] G. G. Kozlov, Spectrum and eigen functions of the operator $H_{U} f(x) \equiv f(U-1 / x) / x^{2}$ and strange attractor's density for the mapping $x_{n+1}=1 /\left(U-x_{n}\right)$, arXiv: 0803.1920. 